\title{
The group inverse of a product*
}

\author{
Xavier Mary ${ }^{a}$, Pedro Patrício ${ }^{b}$
}

\begin{abstract}
${ }^{a}$ Université Paris-Ouest Nanterre - La Défense, Laboratoire Modal'X, 200 avenue de la république, 92000 Nanterre, France. email: xavier.mary@u-paris10.fr

${ }^{b}$ CMAT - Centro de Matemática and Departamento de Matemática e Aplicações , Universidade do Minho, 4710-057 Braga, Portugal. email: pedro@math.uminho.pt
\end{abstract}

\begin{abstract}
In this paper, we characterize the existence and give an expression of the group inverse of a product of two regular elements by means of a ring unit.
\end{abstract}

Keywords: Generalized inverse, group inverse, von Neumann inverse, rings

AMS classification: 15A09, 16E50

\section{Introduction}

In this paper, we consider elements on a general (associative) ring $R$ with unity 1 . We will follow the standard notation regarding generalized inverses. We say $a$ is regular if $a \in a R a$. In this case, a particular solution to $a x a=a$, called von Neumann inverse of $a$, is denoted by $a^{-}$. A reflexive inverse of $a$, denoted by $a^{+}$, is a common solution to $a x a=a, x=x a x$. A regular element $a$ has a reflexive $a^{+}$, namely $a^{-} a a^{=}$, for any choice of von Neumann inverses $a^{-}, a^{=}$.

We say $a$ is group invertible if there is a common solution to $a x a=a, x a x=x, a x=x a$. It is well known that such a solution is unique in case it exists. It is denoted by $a^{\#}$.

Our main goal is to characterize the group inverse of a product of regular elements, as well as to derive an expression of such a group inverse that does not rely on the knowledge of von Neumann regularity of the product.

*This research was financed by Laboratoire Modal'X, Université Paris-Ouest, and by the Research Centre of Mathematics of the University of Minho with the Portuguese Funds from the "Fundação para a Ciência e a Tecnologia", through the Project PEst-OE/MAT/UI0013/2014. 


\section{Main result}

Let $a, b$ be regular elements in $R$, with reflexive inverses $a^{+}, b^{+}$, respectively. Let also

$$
w=\left(1-b b^{+}\right)\left(1-a^{+} a\right)
$$

which we will assume to be regular in $R$.

Note that the regularity of $w$ does not depend on the choices of $a^{+}$and $b^{+}$. That is to say, if $w$ is regular for a particular choice of $a^{+}$and of $b^{+}$, then it must be regular for all choices of $a^{+}$and $b^{+}$. This can be easily proved by noting that $w$ being regular is equivalent to the regularity of the matrix $\left[\begin{array}{ll}a & 0 \\ 1 & b\end{array}\right]$, using [6], which it turn is equivalent to $\left(1-b b^{=}\right)\left(1-a^{=} a\right)$ being regular, for any other choices of von Neumann inverses $a^{=}$and $b^{=}$of $a$ and $b$.

Consider the matrix $M=\left[\begin{array}{cc}a b & a \\ 0 & 1\end{array}\right]=A Q$ with $A=\left[\begin{array}{cc}a & 0 \\ 1 & -b\end{array}\right], Q=\left[\begin{array}{ll}b & 1 \\ 1 & 0\end{array}\right]$.

It is well known that $M^{\#}$ exists if and only if $(a b)^{\#}$ exists, using [1]. Furthermore, the $(1,1)$ entry of $M^{\#}$ equals $(a b)^{\#}$. Also, $M^{\#}$ exists if and only if $U=A Q+I-A A^{-}$is invertible, see [7], [5], in which case $(A Q)^{\#}=U^{-2}(A Q)$.

As $A Q+I-A A^{-}=A\left(Q-A^{-}\right)+I$ then $A Q+I-A A^{-}$is invertible if and only if $\left(Q-A^{-}\right) A+I=Q A+I-A^{-} A$ is invertible, using Jacobson's Lemma, which in turn means $(Q A)^{\#}$ exists. Therefore, by considering the matrix $W=Q A=\left[\begin{array}{cc}b a+1 & -b \\ a & 0\end{array}\right]$, then $(a b)^{\#}$ exists if and only if $W$ is group invertible.

Using [4], the matrix $W$ is group invertible if and only if

$$
\begin{aligned}
z & =(1+b a)\left(1-a^{+} a\right)+b a+\left(1-w w^{-}\right)\left(1-b b^{+}\right)(1+b a) \\
& =1-a^{+} a+b a+\left(1-w w^{-}\right)\left(1-b b^{+}\right)
\end{aligned}
$$

is a unit.

We have, hence, the equivalence

$$
(a b)^{\#} \text { exists if and only if } 1-a^{+} a+b a+\left(1-w w^{-}\right)\left(1-b b^{+}\right) \text {is a unit. }
$$

Using the expression presented in [4] does not give a tratable algorithm to actually compute $(a b)^{\#}$. We will, therefore, pursue a different strategy and compute the $(1,1)$ entry of $M^{\#}$.

Recall that for $M=A Q$ and $Q$ invertible, the group inverse of $M$ exists if and only if $U=A Q+I-A A^{-}$is invertible. For $A=\left[\begin{array}{cc}a & 0 \\ 1 & -b\end{array}\right]$, there exists $A^{-}$for which

$$
A A^{-}=\left[\begin{array}{cc}
a a^{+} & 0 \\
-\left(1-w w^{-}\right)\left(1-b b^{+}\right) a^{+} & b b^{+}+w w^{-}\left(1-b b^{+}\right)
\end{array}\right]
$$

using [6]. 
The matrix $U$ then becomes

$$
U=\left[\begin{array}{cc}
a b+1-a a^{+} & a \\
\left(1-w w^{-}\right)\left(1-b b^{+}\right) a^{+} & 2-b b^{+}-w w^{-}\left(1-b b^{+}\right)
\end{array}\right] .
$$

Multiplication on the right by $K=\left[\begin{array}{cc}1 & 0 \\ a^{+}-b & 1\end{array}\right]$ gives

$$
G=\left[\begin{array}{cc}
1 & a \\
\alpha & 2-b b^{+}-w w^{-}\left(1-b b^{+}\right)
\end{array}\right]
$$

where

$$
\begin{aligned}
\alpha & =\left(1-w w^{-}\right)\left(1-b b^{+}\right) a^{+}+\left(2-b b^{+}-w w^{-}\left(1-b b^{+}\right)\right)\left(a^{+}-b\right) \\
& =a^{+}-b+2\left(1-w w^{-}\right)\left(1-b b^{+}\right) a^{+},
\end{aligned}
$$

as $\left(1-b b^{+}\right) b=0$.

We are left with showing when is $G$ invertible. We do so using the Schur complement on the $(1,1)$ entry. This Schur complement equals

$$
\begin{aligned}
G / I= & \left(2-b b^{+}-w w^{-}\left(1-b b^{+}\right)\right)-\left(\left(1-w w^{-}\right)\left(1-b b^{+}\right) a^{+}+\right. \\
& \left.+\left(2-b b^{+}-w w^{-}\left(1-b b^{+}\right)\right)\left(a^{+}-b\right)\right) a \\
= & \left(2-b b^{+}-w w^{-}\left(1-b b^{+}\right)\right)\left(1-a^{+} a+b a\right)-\left(1-w w^{-}\right)\left(1-b b^{+}\right) a^{+} a \\
= & \left(1+\left(1-w w^{-}\right)\left(1-b b^{+}\right)\right)\left(1-a^{+} a+b a\right)-\left(1-w w^{-}\right)\left(1-b b^{+}\right) a^{+} a \\
= & 1-a^{+} a+b a+\left(1-w w^{-}\right)\left(1-b b^{+}\right)\left(1-2 a^{+} a\right) \\
= & 1-a^{+} a+b a+\left(1-w w^{-}\right)\left(1-b b^{+}\right)\left(1-a^{+} a\right)+ \\
& +\left(1-w w^{-}\right)\left(1-b b^{+}\right) a^{+} a \\
= & 1-a^{+} a+b a+\left(1-w w^{-}\right)\left(1-b b^{+}\right)
\end{aligned}
$$

This gives, and as previously shown,

$(a b)^{\#}$ exists if and only if $z=1-a^{+} a+b a+\left(1-w w^{-}\right)\left(1-b b^{+}\right)$is a unit.

As a side note, we construct another unit associated with $z$, namely we may show that $z=1-a^{+} a+b a+\left(1-w w^{-}\right)\left(1-b b^{+}\right)$is a unit if and only if $z^{\prime}=1-a a^{+}+a b-a(1-$ $\left.w w^{-}\right)\left(1-b b^{+}\right) a^{+}$is a unit. This follows by the sequence of identities $\left(1-w w^{-}\right)\left(1-b b^{+}\right)=$ $\left(1-w w^{-}\right)\left(1-b b^{+}\right)\left(1-a^{+} a+a^{+} a\right)=\left(1-w w^{-}\right)\left(1-b b^{+}\right) a^{+} a$ together with Jacobson's Lemma.

We remark that given a reflexive inverse $w^{+}$of $w$, the element $\tilde{w}=\left(1-a^{+} a\right) w^{+}\left(1-b b^{+}\right)$is an idempotent reflexive inverse of $w$. As such $z$ and $z^{\prime}$ simplify to $1-a^{+} a+b a+1-b b^{+}-w \tilde{w}$ and $1+a b-a b b^{+} a^{+}-a w \tilde{w} a^{+}$, respectively.

We know, using [5, Corollary 3.3(4)], that $(A Q)^{\#}$ exists if and only if $U$ is invertible, in which case $(A Q)^{\#}=U^{-2}(A Q)$. The matrices $U$ and $G$ are equivalent, and we are able to relate their inverses by means of the matrix $K$. Indeed, since $G=U K$, then $U^{-1}=K G^{-1}$. Firstly, we need to compute the inverse of $G$, for which we will use the following known result: 


\section{Lemma 2.1.}

$$
\left[\begin{array}{ll}
1 & y \\
x & z
\end{array}\right]^{-1}=\left[\begin{array}{cc}
1+y \zeta^{-1} x & -y \zeta^{-1} \\
-\zeta^{-1} x & \zeta^{-1}
\end{array}\right]
$$

where $\zeta=z-y x$ is the Schur complement.

Our purpose is to derive an expression for $(a b)^{\#}$, which equals the $(1,1)$ entry of $M^{\#}$.

The $(1,1)$ entry of $M^{\#}$ is obtained by multiplying the first row of $U^{-2}$ by the first column of $A Q$, which is $\left[\begin{array}{c}a b \\ 0\end{array}\right]$. So, in fact we just need the $(1,1)$ entry of $U^{-2}$, which is then multiplied on the right by $a b$ to give $(a b)^{\#}$.

We recall that $G=U K$, where $K=\left[\begin{array}{cc}1 & 0 \\ a^{+}-b & 1\end{array}\right]$, which gives $U^{-1}=K G^{-1}$ and $U^{-2}=K G^{-1} K G^{-1}$. Pre-multiplication with $K$ does not afect the first row, and so we just need the $(1,1)$ element of $G^{-1} K G^{-1}$. Calculations show that

$$
U^{-2}=\left(K G^{-1}\right)^{-2}=\left[\begin{array}{cc}
\left(1+a z^{-1} \alpha\right)^{2}-a z^{-1}\left(a^{+}-b\right)\left(1+a z^{-1} \alpha\right)+a z^{-2} \alpha & ? \\
? & ?
\end{array}\right]
$$

We will need the simplification

$$
b-z b=a^{+} a b-b a b,
$$

from where we obtain

$$
\begin{aligned}
& \alpha a b=\left(a^{+}-b\right) a b=b-z b \\
& \left(1+a z^{-1} \alpha\right) a b=a z^{-1} b .
\end{aligned}
$$

Indeed, $\alpha a b=a a^{+} a b-b a b+2\left(1-w w^{-}\right)\left(1-b b^{+}\right) a^{+} a b$ whose last summand can be expressed as $2\left(1-w w^{-}\right)\left(1-b b^{+}\right) a^{+} a b=-2\left(1-w w^{-}\right)\left(1-b b^{+}\right)\left(1-a^{+} a-1\right) b=-2\left(1-w w^{-}\right) w+2(1-$ $\left.w w^{-}\right)\left(1-b b^{+}\right) b=0$, and therefore $\alpha a b=a^{+} a b-b a b$.

Therefore,

$$
(A Q)^{\#}=\left[\begin{array}{cc}
(a b)^{\#} & ? \\
0 & ?
\end{array}\right]=\left[\begin{array}{cc}
\left(\left(1+a z^{-1} \alpha\right)^{2}-a z^{-1}\left(a^{+}-b\right)\left(1+a z^{-1} \alpha\right)+a z^{-2} \alpha\right) a b & ? \\
0 & ?
\end{array}\right]
$$

from which we obtain the general formula

$$
\begin{aligned}
(a b)^{\#}= & \left(\left(1+a z^{-1} \alpha\right)^{2}-a z^{-1}\left(a^{+}-b\right)\left(1+a z^{-1} \alpha\right)+a z^{-2} \alpha\right) a b \\
= & a b+2\left(a z^{-1} b-a b\right)+a z^{-1} \alpha\left(a z^{-1} b-a b\right)- \\
& a z^{-1}\left(a^{+}-b\right) a z^{-1} b+a z^{-1}\left(z^{-1} b-b\right) \\
= & \left(a z^{-1} \alpha a z^{-1} b-a z^{-1}\left(a^{+}-b\right) a z^{-1} b\right)+a z^{-2} b \\
= & 2 a z^{-1}\left(1-w w^{-}\right)\left(1-b b^{+}\right) z^{-1} b+a z^{-2} b \\
= & 2 a z^{-1} b-2\left(a z^{-1} b\right)^{2}+a z^{-2} b .
\end{aligned}
$$


From $1-a^{+} a=z^{-1}-z^{-1} a^{+} a$ we obtain, by post-multiplying by $b$,

$$
b-a^{+} a b=z^{-1} b-z^{-1} a^{+} a b
$$

which implies

$$
a z^{-1} b=a z^{-1} a^{+} a b .
$$

Now, from (1) we have $z^{-1} b=b+z^{-1} a^{+} a b-z^{-1} b a b$ which implies, using (2), that

$$
z^{-1} b a b=a^{+} a b
$$

which in turns delivers

$$
a b=a z^{-1} b a b .
$$

Using (4) and (5), together with $(a b)^{\#}=2 a z^{-1} b-2\left(a z^{-1} b\right)^{2}+a z^{-2} b$, we write the idempotent $(a b)^{\#}(a b)$ as

$$
\begin{aligned}
(a b)^{\#} a b & =2 a z^{-1} b-2\left(a z^{-1} b\right)^{2}+a z^{-2} b \\
& =2 a z^{-1} b a b-2 a z^{-1} b a b+a z^{-1} z^{-1} b a b \\
& =a z^{-1} a^{+} a b
\end{aligned}
$$

Using (3), this equals $a z^{-1} b$ and therefore $a z^{-1} b$ is an idempotent, the unit of the group generated by $a b$. This simplifies the expression of $(a b)^{\#}$ to

$$
(a b)^{\#}=a z^{-2} b .
$$

It comes with no surprise that the expression of $(a b)^{\#}$ is of the form $a X b$, for a suitable $X$.

We have, from the above, our main result:

Theorem 2.2. Let $a, b$ be regular elements in $R$ with reflexive inverses $a^{+}$and $b^{+}$, respectively. Assume, also, that $w=\left(1-b b^{+}\right)\left(1-a^{+} a\right)$ is regular. Then $(a b)^{\#}$ exists if and only if $z=1-a^{+} a+b a+\left(1-w w^{-}\right)\left(1-b b^{+}\right)$is a unit. In this case,

$$
(a b)^{\#}=a z^{-2} b .
$$

\section{Special cases}

\section{On $a g$ where $g$ is a unit}

We consider a special instance of a product, where one element is a unit. Precisely, let $a, g \in R$ with $g$ a unit. In [5, Corollary 3.2], the existence of the group inverse of $a g$ was related to the existence of a unit, and an expression of $(a g)^{\#}$ was given. By Theorem 2.2, we know that 
$(a g)^{\#}$ exists if and only if $z=1-a^{+} a+g a+\left(1-w w^{-}\right)\left(1-g g^{+}\right)$is a unit. But $g^{+}=g^{-1}$ so that $(a g)^{\#}$ exists if and only if $z=1-a^{+} a+g a$ is a unit, which is equivalent to the criterion of [5, Corollary 3.2] by Jacobson's Lemma. However, Theorem 2.2 gives us

$$
(a g)^{\#}=a\left(1-a^{+} a+g a\right)^{-2} g
$$

which is different from [5, Corollary 3.2]. On the other hand, we can also consider the case of a group inverse of $g a$. In this case the $w$ in the previous theorems is 0 . $(g a)^{\#}$ exists if and only if $a g-1+a a^{+}$is a unit, and

$$
(g a)^{\#}=g\left(a g-1+a a^{+}\right)^{-2} a .
$$

As the existence of $(a g)^{\#}$ is equivalent with the existence of $(g a)^{\#}$ (and using $-g$ instead of $g$ in the second case), we get:

Corollary 3.1. The following conditions are equivalent:

1. ag is group invertible;

2. ga is group invertible;

3. $z=1-a^{+} a+g a$ is a unit;

4. $\eta=1-a a^{+}+a g$ is a unit;

In which case:

$$
(a g)^{\#}=a z^{-2} g \text { and }(g a)^{\#}=g \eta^{-2} a .
$$

The units in the previous corollary are strongly related to the existence of the inverse of $g$ along $a$, see [3].

As a special case, when $g=1$, we recover the classical result ([5, Corollary 3.3.]):

Corollary 3.2. The following conditions are equivalent:

1. a is group invertible;

2. $z=1-a^{+} a+a$ is a unit;

3. $\eta=1-a a^{+}+a$ is a unit;

In which case:

$$
(a)^{\#}=a z^{-2} \text { and }(a)^{\#}=\eta^{-2} a \text {. }
$$




\section{On the sum}

We now apply the results of the previous section to the sum of ring elements to obtain a known characterization of the group inverse of a sum.

Let $a, b$ be ring elements such that $a+b$ is regular. Consider $A=\left[\begin{array}{ll}a & 1 \\ 0 & 0\end{array}\right]$ and $B=\left[\begin{array}{ll}1 & 0 \\ b & 0\end{array}\right]$, for which $A B=\left[\begin{array}{cc}a+b & 0 \\ 0 & 0\end{array}\right]$. This is a key factorization that allows us to characterize the group inverse of $a+b$ by the group inverse of $A B$.

Using the results on the previous section, $(A B)^{\#}$ exists if and only if $H=I-A^{+} A+$ $B A-\left(I-W W^{-}\right)\left(I-B B^{+}\right)$is invertible. We will now undertake the computation of this matrix, for particular choices of inner and reflexive inverses.

We will take $A^{+}=\left[\begin{array}{ll}0 & 0 \\ 1 & 0\end{array}\right]$ and $B^{+}=\left[\begin{array}{ll}1 & 0 \\ 0 & 0\end{array}\right]$, which will deliver $A^{+} A=\left[\begin{array}{ll}0 & 0 \\ a & 1\end{array}\right]$ and $B B^{+}=\left[\begin{array}{cc}1 & 0 \\ b & 0\end{array}\right]$.

Also, $B A=\left[\begin{array}{cc}a & 1 \\ b a & b\end{array}\right]$ and $W=\left(I-B B^{+}\right)\left(I-A^{+} A\right)=\left[\begin{array}{cc}0 & 0 \\ -a-b & 0\end{array}\right]$. Since $a+b$ is regular, then $W$ is regular and we may take $W^{-}=\left[\begin{array}{cc}0 & (-a-b)^{-} \\ 0 & 0\end{array}\right]$. The associated idempotents take the form $W W^{-}=\left[\begin{array}{cc}0 & 0 \\ 0 & (a+b)(a+b)^{-}\end{array}\right]$and $I-W W^{-}=\left[\begin{array}{cc}1 & 0 \\ 0 & 1-(a+b)(a+b)^{-}\end{array}\right]$.

We therefore obtain

$$
\left(I-W W^{-}\right)\left(I-B B^{+}\right)=\left[\begin{array}{cc}
0 & 0 \\
-\left(1-(a+b)(a+b)^{-}\right) b & 1-(a+b)(a+b)^{+}
\end{array}\right] .
$$

The invertible matrix takes the form

$$
\left[\begin{array}{cc}
1+a & 1 \\
-a+b a+1\left(1-(a+b)(a+b)^{+}\right) b & b-1+(a+b)(a+b)^{+}
\end{array}\right]
$$

whose Schur complement equals $(a+b)+1-(a+b)^{+}(a+b)$ and which has to be a ring unit. This is coherent with the known result.

\section{The example of a trace product}

Let $a, b$ be two elements of the ring $R$ such that $R a b=R b$ and $a b R=a R$ (one says that $a b$ is a trace product). Then it is known, by a theorem of Clifford ([2, Proposition 2.3.7]), that this is equivalent with the existence of an idempotent $e \in R$ such that $R a=R e$ and $b R=e R$. In particular, $a$ and $b$ are von Neumann regular and we can find $a^{+}$and $b^{+}$such that $a^{+} a=b b^{+}=e$. For this particular choice, $w=\left(1-b b^{+}\right)\left(1-a^{+} a\right)=(1-e)$ is idempotent, and the element $z=1-a^{+} a+b a+\left(1-w w^{-}\right)\left(1-b b^{+}\right)$reduces to $z=1-a^{+} a+b a$. As the 
invertibility of $z$ implies the invertibility of all the elements of the form $1-a^{+} a+b a$, we get by Theorem 2.2 :

Corollary 3.3. Let $a b$ be a trace product. Then $a$ and $b$ are regular, and for any choice of $a^{+}, a b$ is group invertible if and only if $z=1-a^{+} a+b a$ is a unit. In this case,

$$
(a b)^{\#}=a\left(1-a^{+} a+b a\right)^{-2} b .
$$

Once again, we recognize the criterion of invertibility of $b$ along $a$.

\section{Final remarks}

1. In this paper, we are primarly interested in the group invertibility (on the group inverse) of the product $a b$. Since the group inverse of $a b$ equals $a z^{-2} b$, what can be said about $b a ?$

If $z^{-1}$ is an inner inverse, and since $a b$ is group invertible, then $1+a b-(a b)(a b)^{\#}$ is a unit, that is $1+a b-a z^{-1} b$ is a unit. By Jacobson lemma, $1+b a-b a z^{-1}$ is a unit, and if $z^{-1}$ is an inner inverse of $b a$, then $b a$ is actually group invertible.

Let us compute $z\left(b a z^{-1} b a-b a\right)$.

$$
\begin{aligned}
z\left(b a z^{-1} b a-b a\right) & =\left(1-a^{+} a+b a+\left(1-w w^{-}\right)\left(1-b b^{+}\right)\right)\left(b a z^{-1} b a-b a\right) \\
& =\left(1-a^{+} a+b a\right)\left(b a z^{-1} b a-b a\right) \text { as }\left(1-b b^{+}\right) b=0 \\
& =\left(b a z^{-1} b a-b a\right)+\left(-a^{+}+b\right)\left(a b a z^{-1} b a-a b a\right)
\end{aligned}
$$

But equation (5) gives $a b=a z^{-1} b a b$ and since we have shown that $a z^{-1} b=a b(a b)^{\#}$, then it commutes with $a b$ and $a b=a b a z^{-1} b$. This gives that the second term in the above sum is 0 , and $z\left(b a z^{-1} b a-b a\right)=\left(b a z^{-1} b a-b a\right)$, or equivalently, $(1-z)\left(b a z^{-1} b a-\right.$ $b a)=0$. Multiplying by $z^{-1}$ on the left gives $\left(b a z^{-1} b a-b a\right)=z^{-1} b a z^{-1} b a-z^{-1} b a$ or

$$
\left(z^{-1}-1\right)\left(b a z^{-1} b a-b a\right)=0
$$

Note that $1-z^{-1}$ is a unit if and only if $1-z$ is a unit. Indeed, setting $\chi=1-z$ then $1=z^{-1}-z^{-1} \chi=z^{-1}-\chi z^{-1}$ which imply $z^{-1}-1=z^{-1} \chi=\chi z^{-1}$ and $\chi=1-z$ is a unit. Conversely, if $\chi=1-z$ is a unit then $z^{-1}-1=z^{-1} \chi$ we obtain the desired conclusion.

Suppose $1-z$ is a unit. Then $z^{-1}$ is a inner inverse of $b a$ (and $z^{-1} b a$ is idempotent). Of course, if $z^{-1} b a$ is idempotent then $z^{-1}$ is always an inner inverse of $b a\left(b a=z z^{-1} b a=\right.$ $z z^{-1} b a z^{-1} b a=b a z^{-1} b a$.) 
2. Consider $\left(G_{z},.\right)$ the group generated by $z$, and $\left(G_{a b},.\right)$ the group generated by $a b$. Then we can prove that $\phi:\left(G_{z},.\right) \rightarrow\left(G_{a b},.\right)$ defined by $\phi\left(z^{k}\right)=a z^{k-1} b$ is an isomorphism, or put in an other form: $a z^{n} b=(a b)^{n+1}$ for all $n \in \mathbb{Z}$.

This can be proved by induction on $\mathbb{Z}$. First, we have proved in our paper that $z(b-$ $\left.a^{+} a b\right)=b-a^{+} a b$ and $z^{-1} b a b=a^{+} a b$. By induction on $\mathbb{Z}$, this imply that $z^{n}\left(b-a^{+} a b\right)=$ $b-a^{+} a b$ for all $n \in \mathbb{Z}$ hence $a z^{n} b=a z^{n} a^{+} a b=a z^{n-1} b a b$ for all $n \in \mathbb{Z}$. Now our equation is true for $n=0$. Assume it is true for $n \in \mathbb{N}$. Then $a z^{n+1} b=a z^{n} b a b=(a b)^{n+1}$. Suppose now it is true for $-n \in \mathbb{N}$, that is $a z^{-n} b=(a b)^{1-n}$. Then we prove that $a z^{-n-1} b$ is the group inverse of $(a b)^{n}$. We have $a z^{-n-1} b(a b)^{n}=a z^{-n-1} b(a b)(a b)^{n-1}=$ $a z^{n} b(a b)^{n-1}=(a b)^{0}$. As symmetrically, $(a b)^{n} a z^{-n-1} b=(a b)^{0}$, then $a z^{-n-1} b$ is the group inverse of $(a b)^{n}$.

3. We can compute other units by duality, using the opposite ring $(R, \times), x \times y=y x$. Precisely, $a b$ is group invertible in $(R,$.$) if and only if b \times a$ is group invertible in $(R, \times)$, and by our theorem this happens if and only if $1-b^{+} \times b+a \times b+\left(1-w \times w^{-}\right) \times\left(1-a \times a^{+}\right)$ (if $a^{+}$and $b^{+}$are inverses in $(R,$.$\left.) then they are also inverses in (R, \times)\right)$, with $w=$ $\left(1-a \times a^{+}\right) \times\left(1-b^{+} \times b\right)$, or equivalently if and only if $t=1-b b^{+}+b a+\left(1-a^{+} a\right)\left(1-w^{-} w\right)$ is a unit with $w=\left(1-b b^{+}\right)\left(1-a^{+} a\right)$ (classical one). Note that this unit is a priori different from the other ones in the paper. If we continue the duality principle, we end with $b \times t^{-2} \times a$ is the group inverse of $b \times a$, that is $a t^{-2} b$ is the group inverse of $a b$, so that this unit works equivalently as our $z$.

\section{Acknowledgment}

This research was developed when the second author visited Laboratoire Modal'X, at Université Paris-Ouest. The authors gratefully acknowledge the support of Laboratoire Modal'X.

\section{References}

[1] Hartwig, R. E.; Shoaf, J.; Group inverses and Drazin inverses of bidiagonal and triangular Toeplitz matrices. J. Austral. Math. Soc. Ser. A, 24 (1977), no. 1, 10-34.

[2] Howie J.M.; Fundamentals of Semigroup Theory. London Mathematical Society Monographs. New Series, 12. Oxford Science Publications, 1995.

[3] Mary, X., Patrício, P., Generalized inverses modulo $\mathcal{H}$ in semigroups and rings, Linear and Multilinear Algebra, 61 (2013), no. 8, 1130-1135.

[4] Patrício, P.; Hartwig, R.E; The (2,2,0) Group Inverse Problem, Applied Mathematics and Computation, 217(2) (2010), 516-520. 
[5] Patrício, P.; Hartwig, R.E.; Some regular sums. Linear and Multilinear Algebra, 63(1) (2015), 185-200.

[6] Patrício, P.; Puystjens, R.; About the von Neumann regularity of triangular block matrices. Linear Algebra Appl. 332/334 (2001), 485-502.

[7] Puystjens, R.; Hartwig, R. E.; The group inverse of a companion matrix. Linear and Multilinear Algebra, 43 (1997), no. 1-3, 137-150, 